\title{
MAJORIZING SEQUENCES FOR NEWTON'S METHOD
}

\author{
J. M. GUTIÉRREZ AND M. A. HERNÁNDEZ
}

\begin{abstract}
Majorizing sequences for Newton's method are analysed from a new standpoint. As a consequence, we give convergence results under assumptions different from the classical Kantorovich conditions.
\end{abstract}

One of the most known techniques for studying a sequence $\left\{x_{n}\right\}$ defined in Banach spaces is majorizing theory. In brief, consider an equation $x=G(x)$, where $G$ is an operator defined on the ball $\left\|x-x_{0}\right\|<R$ in some Banach space $X$. Take also the real equation $t=g(t)$, where $g$ is defined in the interval $\left[a_{0}, a_{1}\right],\left(a_{1}-a_{0}<R\right)$. We shall say that equation $t=g(t)$ (or function $g$ ) majorizes equation $x=G(x)$ (or operator $G$ ) if

$$
\begin{array}{r}
\left\|G\left(x_{0}\right)-x_{0}\right\| \leq g\left(t_{0}\right)-t_{0}, \quad t_{0} \in\left[a_{0}, a_{1}\right] \\
\left\|G^{\prime}(x)\right\| \leq g^{\prime}(x), \quad \text { whenever }\left\|x-x_{0}\right\| \leq t-t_{0} .
\end{array}
$$

In this situation, the convergence of the iterative process $x_{n+1}=G\left(x_{n}\right)$ in $X$ can be deduced from the one of the iteration $t_{n+1}=g\left(t_{n}\right)$ on the real line [1], [2].

For solving a nonlinear operator equation

$$
F(x)=0
$$

we can consider Newton's method:

$$
x_{n+1}=G\left(x_{n}\right), \quad G(x)=x-F^{\prime}(x)^{-1} F(x) .
$$

Here $F$ is an operator defined from a Banach space $X$ into another Banach space $Y$. Following Kantorovich [1], we shall assume that $F$ is defined and has a continuous second derivative in a closed ball $\Omega_{0}=\left\{x \in X ;\left\|x-x_{0}\right\| \leq R_{0}\right\}$. Assume, in addition, that

(i) $\Gamma_{0}=F^{\prime}\left(x_{0}\right)^{-1}$ exists and is a continuous linear operator,

(ii) $\left\|\Gamma_{0} F\left(x_{0}\right)\right\| \leq a$,

(iii) $\left\|\Gamma_{0} F^{\prime \prime}(x)\right\| \leq b, x \in \Omega_{0}$.

Received September 18, 1997, Revised February 12, 1998.

1991 Mathematics Subject Classification. Primary 47H10, Secondary 65J15.

Key words and phrases. Nonlinear equations in Banach spaces, Newtọn's method, majorizing sequences.

Supported in part by a grant of the University of La Rioja (ref. API 98/B12). 
Then if $h=a b \leq 1 / 2$, and $(1-\sqrt{1-2 h}) / b \leq R_{0}$, the equation (3) has a solution $x^{*}$ and the sequence $\left\{x_{n}\right\}$ defined in (4) converges to $x^{*}$. Additional information on uniqueness of solution and error bounds can be seen in [1], [3].

The convergence of the sequence $\left\{x_{n}\right\}$ follows from the convergence of the real sequence

$$
t_{n+1}=t_{n}-\frac{p\left(t_{n}\right)}{p^{\prime}\left(t_{n}\right)}, \quad t_{0}=0
$$

where

$$
p(t)=\frac{b}{2} t^{2}-t+a
$$

On the other hand, in [4] it was shown that the derivative of $G$ defined in (4) is the linear operator $L_{F}(x): X \rightarrow X$ given by

$$
L_{F}(x)=F^{\prime}(x)^{-1} F^{\prime \prime}(x) F^{\prime}(x)^{-1} F(x) .
$$

Now we are going to study Newton sequence in terms of this linear operator $L_{F}(x)$ and its correspondent real expression.

Notice that condition (1) tells us $\left\|x_{1}-x_{0}\right\|=\left\|\Gamma_{0} F\left(x_{0}\right)\right\| \leq a=t_{1}-t_{0}$. Besides, if $x \in\left[x_{0}, x_{1}\right]$, i.e., $x=x_{0}+s\left(x_{1}-x_{0}\right), 0 \leq s \leq 1$, then we have $\left\|L_{F}(x)\right\| \leq L_{p}(t)$ where $t \in\left[t_{0}, t_{1}\right]$. Indeed,

$$
\begin{aligned}
\Gamma_{0} F(x) & =\Gamma_{0} F\left(x_{0}\right)+\left(x-x_{0}\right)+\int_{x_{0}}^{x} \Gamma_{0} F^{\prime \prime}(z)(x-z) d z \\
& =\Gamma_{0} F\left(x_{0}\right)(1-s)+\int_{x_{0}}^{x} \Gamma_{0} F^{\prime \prime}(z)(x-z) d z
\end{aligned}
$$

Then

$$
\left\|\Gamma_{0} F(x)\right\| \leq(1-s) a+\frac{b}{2} s^{2} a^{2}=a-t+\frac{b}{2} t^{2},
$$

where $t=s a \in\left[0, t_{1}\right]$.

Following a standard technique and using Banach lemma on invertion of operators [3], we have

$$
\left\|\left[\Gamma_{0} F^{\prime}(x)\right]^{-1}\right\| \leq \frac{1}{1-b\left\|x-x_{0}\right\|} \leq \frac{1}{1-b t} .
$$

Then

$$
\left\|L_{F}(x)\right\| \leq \frac{b\left(a-t+(b / 2) t^{2}\right)}{(1-b t)^{2}}=L_{p}(t)
$$

and therefore

$$
\left\|x_{2}-x_{1}\right\|=\left\|G\left(x_{1}\right)-G\left(x_{0}\right)\right\|=\left\|\int_{x_{0}}^{x_{1}} L_{F}(x) d x\right\| \leq \int_{t_{0}}^{t_{1}} L_{p}(t) d t=t_{2}-t_{1} .
$$

In general, we can replace $x_{0}$ by $x_{1}, x_{1}$ by $x_{2}$ and so on, obtaining $\left\|L_{F}(x)\right\| \leq L_{p}(t)$ for $x \in\left[x_{n}, x_{n+1}\right]$ and $t \in\left[t_{n}, t_{n+1}\right]$. What we have just proved is that, under $\mathbf{K a n t o r o v i c h}$ assumptions, conditions (1) and (2) are fulfilled. 
Now we are going to follow the inverse reasoning, that is, assume there exists a function $\omega(t)$ satisfying $\left\|L_{f}(x)\right\| \leq \omega(t)$. If a function $f$ is such that $L_{f}(t)=\omega(t)$, we can use it to prove the convergence of (4). Under Kantorovich assumptions we have just seen $\omega(t)=L_{p}(t)$, where $p(t)$ is the polynomial given by (5). We wonder now, if we have another function $\omega(t)$, can we obtain convergence conditions different from the ones of Kantorovich? The answer is affirmative, as we see next.

Let us consider, for instance, the situation $\left\|L_{F}(x)\right\| \leq M<1, x \in \overline{B\left(x_{0}, R_{0}\right)}$. Functions $f$ with a positive root $r_{1}$ and satisfying $L_{f}(t)=M$ have the form

$$
f(t)=\left(r_{1}-t\right)^{1 /(1-M)} .
$$

Notice that this kind of functions are decreasing and convex in the interval $\left[0, r_{1}\right]$. Then, Newton's method for solving $f(t)=0$ is convergent starting at any point of $\left[0, r_{1}\right]$ (see [5]).

If $t_{0}=0$. and $\left\|\Gamma_{0} F\left(x_{0}\right)\right\| \leq a$, then condition (1) holds if and only if $a \leq$ $-f\left(t_{0}\right) / f^{\prime}\left(t_{0}\right)=r_{1}(1-M)$, that is, $r_{1} \geq a /(1-M)$.

Finally, in order to assure that Newton sequence lies in the ball $\overline{B\left(x_{0}, R_{0}\right)}$ we have to require $r_{1} \leq R_{0}$. So we have just proved the following result.

Theorem 1. Let $F(x)=0$ be an equation, where $F$ is an operator defined between two Banach spaces $X$ and $Y$. Assume that $F$ satisfies $\left\|L_{F}(x)\right\| \leq M<1, x \in \overline{B\left(x_{0}, R_{0}\right)}$. If $\left\|\Gamma_{0} F\left(x_{0}\right)\right\| \leq a$ and $a /(1-M) \leq R_{0}$, then the Newton sequence

$$
t_{n+1}=t_{n}-\frac{f\left(t_{n}\right)}{f^{\prime}\left(t_{n}\right)}, \quad t_{0}=0
$$

where $f$ is given by (6), majorizes the sequence $\left\{x_{n}\right\}$ given by (4). Besides, as $\left\{t_{n}\right\}$ converges to $r_{1},\left\{x_{n}\right\}$ is also convergent, and the limit $x^{*}$ is a solution of (3).

Notice that the above.result is more general than Kantorovich theorem. Just taking the scalar function $f(t)=(1-t)^{4}$ we have $L_{f}(t)=3 / 4$ for all $t \in \mathbb{R}$ (define $L_{f}(1)=$ $\left.\lim _{t \rightarrow 1} L_{f}(t)\right)$. However, Kantorovich conditions are not fulfilled in the interval $[0,1]$. Indeed,

$$
a=-\frac{f(0)}{f^{\prime}(0)}=\frac{1}{4}, \quad b=-\sup _{t \in[0,1]} \frac{f^{\prime \prime}(t)}{f^{\prime}(0)}=3 \quad \text { and } h=a b=\frac{3}{4}>\frac{1}{2} .
$$

On the other hand, $a /(1-M)=1=r_{1}$ and the previous result guarantees the convergence of Newton's method starting at $t_{0}=0$.

By using Theorem 1 we have got a wider domain of starting points for Newton's method. However error estimates are, in general, worse than the ones obtained from polynomial (5). Notice that function (6) has a multiple root and in this situation it is well known [6] that the convergence of Newton's method is linear.

Instead of condition $\left\|L_{F}(x)\right\| \leq M<1$ we can consider other bounds for $\left\|L_{F}(x)\right\|$, not necessarily constants. For instance, if we have

$$
\left\|L_{F}(x)\right\| \leq \alpha(r-t) \text { for }\left\|x-x_{0}\right\| \leq t-t_{0},
$$


where $\alpha r<2$ and $\left\|\Gamma_{0} F\left(x_{0}\right)\right\| \leq a \leq r(2-\alpha r) / 2$, the majorizing function is

$$
f(t)=\frac{r-t}{\beta+t}, \quad \beta=\frac{2}{\alpha}-r .
$$

This kind of conditions have a theorical interest, but in practice it is difficult to find convenient bounds for $\left\|L_{F}(x)\right\|$. Nevertheless, when this situation happens, we have developed a new method to find majorizing sequences. This method allow us to establish convergence under assumptions different from the classical Kantorovich conditions.

\section{References}

[1] L. V. Kantorovich and G. P. Akilov, "Functional Analysis," Pergamon Press, Oxford, 1982.

[2] W. C. Rheinboldt, "A unified convergence theory for a class of iterative process," SIAM J. Numer. Anal., 5(1968), 42-63.

[3] L. B. Rall, "Computational solution of nonlinear operator equations," Robert E. Krieger Publishing Company, Inc., New York, 1979.

[4] J. M. Gutiérrez, M. A. Hernández and M. A. Salanova, "Accesibility of solutions by Newton's method," Inter. J. Computer Math., 57(1995), 239-247.

[5] A. M. Ostrowski, "Solution of equations and systems of equations," Academic Press, New York, 1973.

[6] J. F. Traub, "Iterative methods for solution of equations," Prentice-Hall, New Jersey, 1964.

University of La Rioja, Dpt. Mathematics and Computation., C/Luis de Ulloa S/N, 26004, Logroño, Spain.

(E-mail: jmguti@dmc.unirioja.es; mahernan@dmc.unirioja.es) 